\begin{tabular}{|c|c|}
\hline Title & $\begin{array}{l}\text { Architectures and Technologies for Adaptation of Secured Content in Governed } \\
\text { Multimedia Applications }\end{array}$ \\
\hline Authors & 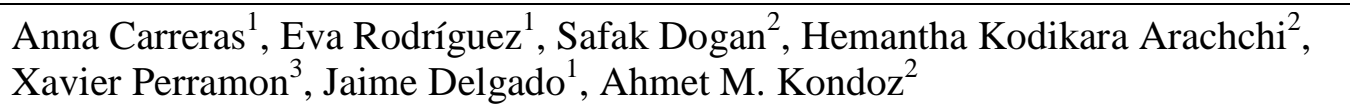 \\
\hline $\begin{array}{l}\text { Affiliations } \\
\text { and } \\
\text { contacts }\end{array}$ & $\begin{array}{l}{ }^{1} \text { Departament d'Arquitectura de Computadors, Universitat Politècnica de } \\
\text { Catalunya, Campus Nord, Mòdul D6, Jordi Girona 1-3, E-08034 Barcelona, Spain } \\
\text { \{annac, evar, jaime.delgado\}@ac.upc.edu } \\
{ }^{2} \text { I-Lab Multimedia Communications Research, Faculty of Engineering and } \\
\text { Physical Sciences, University of Surrey, Guildford, GU2 7XH, Surrey, UK } \\
\{\text { S.Dogan, H.Kodikaraarachchi, A.Kondoz\}@ surrey.ac.uk } \\
\\
{ }^{3} \text { Dept. of Information and Communication Technologies, Universitat Pompeu } \\
\text { Fabra, Roc Boronat 138, E-08018, Barcelona, Spain } \\
\text { xavier.perramon@ upf.edu }\end{array}$ \\
\hline
\end{tabular}




\title{
Architectures and Technologies for Adaptation of Secured Content in Governed Multimedia Applications
}

\begin{abstract}
The wide range of media and consumer devices, currently available, has raised new security requirements for multimedia systems and their applications. Despite the wide variety of work conducted on the access control, Digital Rights Management (DRM) and adaptation areas to date, there is a lack of initiatives addressing them together. This article analyzes existing initiatives and proposes new technologies for the governed adaptations of secured content in heterogeneous environments. These technologies are integrated in an architecture for the secure management of multimedia contents, which covers the complete digital content value chain, from creation to consumption by end users. Video Surveillance is the application scenario chosen for further illustrating the use of the proposed architecture. Two use cases are discussed in order to address governance, protection and adaptation requirements in surveillance applications.
\end{abstract}

Index Terms-Protected content adaptation, digital rights management systems, access control, video surveillance

\section{INTRODUCTION}

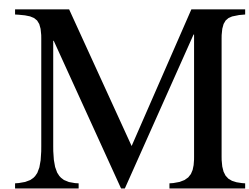

ultimedia applications managing protected content in heterogeneous environments have increased considerably in recent years. Nowadays, consumers and multimedia system users wish to access any kind of content from any device they have available. However, in most cases, resources are not available to all the users of a system, which in turn has to control user access and resource usage and management. On the other hand, if authorized users consume contents in heterogeneous usage environments, the contents need to be adapted for enhancing the users' perceived quality. Therefore, platforms managing digital contents have to provide functionalities for users' authentication, content protection and governance, data integrity and authenticity, content adaptation, content consumption and notification of events in an integrated fashion.

This paper is organized as follows. Section II provides an overview of the state-of-the-art in the adaptation of secured content in governed multimedia applications. Section III proposes an architecture, which covers a complete Digital Rights Management (DRM) system, presenting an innovative approach for the adaptation of secured content. Section IV presents a specific application scenario, namely Video Surveillance, which poses a challenge for addressing users' privacy protection, secure content access, data integrity and authenticity, users' authentication, efficient transmission of video data and communication applications in heterogeneous usage 
environments. Section V presents the necessary interfaces between the security modules of the architecture, focusing on the Video Surveillance scenario, and the content adaptation results. Finally, Section VI concludes the paper and points out future research lines in the area of privacy protection.

\section{STATE-OF-THE-ART IN ADAPTATION OF SECURED CONTENT IN GOVERNED MULTIMEDIA APPLICATIONS}

Current DRM initiatives specify how digital assets can be managed in a controlled way throughout the complete digital value chain. Among the different content life cycle stages, these initiatives focus on content distribution and consumption. The existing DRM systems enable the distribution and consumption of protected digital assets according to the terms stated by their distributors and rights holders [1]. Digital assets are governed by means of licenses expressed according to a Rights Expression Language (REL), while licenses containing rights and obligations are in turn enforced by license based authorization tools. Despite the significant research work conducted on content adaptation to date [2], not many of the current DRM initiatives consider the secure adaptation of digital assets as one of their requirements. A few of those initiatives enable the adaptation of protected content, but not the necessary fine-grained governance of digital assets. By definition, governance embodies the enforcement of rights expressions, which restrict content adaptations according to the terms stated by content creators and rights holders.

\section{A. Adaptation authorization}

As the development of the two aforementioned areas of research has been pursued separately, it seems impossible to govern content adaptations due to their lack of fine-grained descriptions about permissible conversions nowadays. Only very recently the two groups of researchers working on adaptation and DRM started to cooperate in jointly defining approaches and methodologies to combine each other's outcomes into a single framework [3].

While MPEG-21 Digital Item Adaptation (DIA) provides, among other features, the description of fine-grained media conversions by means of the conversion operations' names and parameters, which can be used to define rights expressions to govern adaptation in an interoperable way; MPEG-21 REL data model for a rights expression includes four basic entities, principal, right, resource and condition. In order to follow this model, the MPEG-21 DIA conversion permissions have to be integrated within the Condition element.

Many of the research projects that have been working with MPEG-21 DIA (e.g., DAIDALOS, aceMEDIA, etc) have earmarked this point as possible future work. We have been involved in other projects like AXMEDIS, VISNET II NoE, and the second part of Projecte Integrat, named project Machine, which have also begun to conduct research work in this area. Thus, to the best of our knowledge, we have developed the first real implementation of this new adaptation authorization concept [4]. 


\section{B. Content protection for adaptation}

Context-aware content adaptation is one of the powerful tools to enhance the perceived quality of the multimedia contents consumed in heterogeneous usage environments. To perform content adaptation operations, certain information embedded in the compressed bit streams has to be available to intermediate nodes of a delivery chain. However, classic video encryption technologies presume no third-party access to any information in the encoded video is necessary. Consequently, intermediate nodes have to decrypt the content before performing any adaptation operation. Decrypting at an intermediate node, however, is not allowed in an end-to-end content protection environment. A number of researchers have attempted to address this issue [5],[6].

Our work, as most of the recent literature, focuses on encrypting H.264/Advanced Video Coding (H.264/AVC) and scalability extension of H.264/AVC (H.264/SVC) compatible bit streams. H.264 organises encoded data into Network Abstraction Layer (NAL) units, each of which is encapsulated into a data packet, and they are independently decodable. This feature improves the ability of adapting the encoded video data in a network friendly way. Hence, most of the encryption schemes proposed for H.264 independently treat each NAL unit. A number of H.264/AVC compatible partial ciphering techniques have been proposed by various researchers [7]-[9]. These techniques encrypt a set of selected syntax elements without disturbing the integrity of the bit stream. As a result, the bit stream can be parsed and subsequently decoded without decrypting the encrypted syntax elements even though the visual quality of the decoded video is greatly compromised. Hence, this type of encryption is suitable when the total confidentiality is not essential [10]. Moreover, these techniques do not consider the adaptability since they do not address encrypting scalable video contents. In contrast, the techniques reported in [11]-[13] ([12] will be used throughout this paper) attempt to implement comprehensive security while retaining the in-network adaptability. Naturally, these techniques encrypt the payloads of each NAL unit while keeping the header information plain so that any in-network adaptation node can treat them as if they are not encrypted. To maintain the independency of each encrypted NAL unit, a unique Initialization Vector (IV) is determined for each NAL unit. Going against this norm, the video encryption architecture discussed in [14] derives the IV from a previously encrypted NAL unit. In this way, a dependency is created between encrypted NAL units. The IV generation technique is designed in such a way that the encryption dependencies match with the encoding dependencies amongst the scalability layers. Hence, loss of any NAL unit makes all the dependent NAL units unencryptable. However, this does not pose any problem because all the dependent NAL units of any dropped NAL unit are also dropped in scalable video adaptation.

The above encryption techniques are specific solutions for each coding technique. In contrast, Mukherjee et al. have developed a format independent technique for encrypting scalable video contents [15]. They propose making use of the MPEG-21 DIA Bit stream Syntax Description (BSD) tool to encrypt bit stream segments progressively. Such a encryption structure creates interdependencies amongst segments which negatively affects the error resilience of the video bit 
streams. Thus, this technique is not suitable for video communication applications over lossy channels.

\section{PROPOSED ARCHITECTURE FOR THE ADAPTATION OF SECURED CONTENT IN GOVERNED MULTIMEDIA APPLICATIONS}

Figure 1 presents the functional architecture of the proposed platform. It covers a complete DRM system while permitting the adaptation of secure content. It is based on the Multimedia Information Protection and Management System (MIPAMS) architecture [16]. MIPAMS was devised for the management of multimedia information taking into account DRM and protection. Specifically, the aim of this DRM architecture is to enable the management of multimedia content through the complete content value chain, from content creation to consumption by end users, including adaptation of digital content. A detailed comparison study of MIPAMS with other DRM architectures can be found in [17].

The Governance Server provides functionalities for the creation and storage of licenses, and online license based authorization; it is responsible for performing authorizations of users to perform actions over resources. When a user tries to perform an action over a resource, this module checks if a license has been issued to the user authorizing him to perform the requested operation.

Furthermore, it integrates adaptation authorization functionalities that permit to govern complex content adaptation operations through the use of detailed descriptions of the permitted conversions expressed in MPEG-21 DIA.

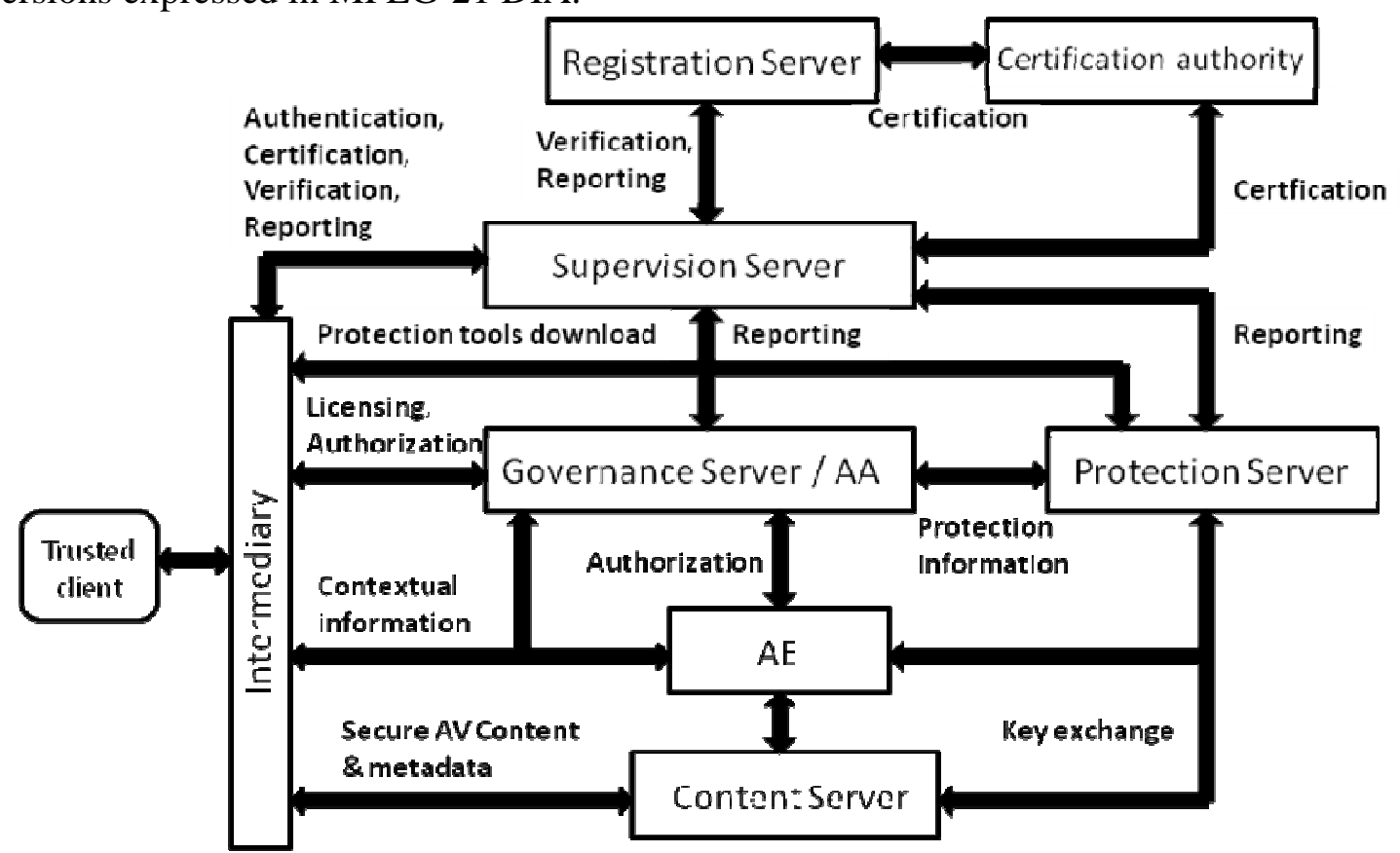

Fig. 1. Proposed architecture for the adaptation of secured content in governed multimedia applications 
The Content Server enables users and applications to create, edit, upload, browse and search digital resources. Those resources can be protected. Then, the protection keys are requested to the Protection Service, which sends an encrypted version of the key to the Content Server, next those keys are resgistered through the Protection Server.

The Protection Server offers a service for protecting digital contents, digital objects or metadata, mainly using encryption techniques and scrambling. The actual data to be protected, either audiovisual media content or metadata, is converted to unintelligible form by means of a scrambling transformation, using a secret key as an input parameter. The values of the scrambling keys are not stored directly in the Protection Server. Instead, an encrypted version of these keys is stored, using an appropriate protection key. The protection keys, and not the scrambling keys, are associated with the corresponding licenses. Both the scrambling of contents and the protection of the scrambling keys are achieved with cryptographic algorithms. The term "scrambling" is simply used to distinguish the encryption of contents from the encryption of keys, for analogy with the traditional use of this term for protected audiovisual media content transmissions.

The Adaptation Engine (AE) is the primary content adaptation module in the proposed architecture. It comprises two main building blocks for performing its adaptation task, namely the adaptation decision taking block and the actual content adapting block. Adaptation decision block is responsible for taking into account all of the usage environment constraints as well as users preferences through the means of various context elements in an adaptation system, and subsequently for processing them and deciding on what kind of a content adaptation tool to be employed to best serve those constraints and preferences. The decision information is then passed to the actual content adapting block, whose task is to execute the necessary tools and operations, as dictated by the taken adaptation decisions. Certainly, both of these tasks are undertaken in line with the necessary authorizations provided by the Governance Server, which also acts as the Adaptation Authorizer (AA) for operation of the overall system.

The proposed architecture is capable of supporting both "trusted" and "untrusted" types of AEs. As the name implies, a trusted AE is the one that can make use of the security keys provided to it for decrypting a secured content, so as to be able to perform a number of both structural and signal-level content adaptation operations on the audiovisual media streams. With a similar analogy, an untrusted $\mathrm{AE}$ is a content adapting engine, with which a security key cannot be shared, and thus the content adaptation operation should be performed on the media stream blindly (that is to say, without decrypting the content) [12].

The Supervision Server provides a number of functionalities, ranging from the authentication and supervision of actors and system components to the receipt and storage of the event reports, regarding content consumption or other relevant issues in the system.

The Registration Server is an equally significant module in this architecture, which is responsible for supplying the necessary certificates to the various actors and components involved in the system in order to guarantee their authenticity and integrity. 
The Intermediary module acts as the interface between the user ("Trusted client" in Figure 1) and the main modules of the platforms. Thus, the user registers, gets authenticated, and selects to access certain contents through this particular module. Furthermore, the Intermediary is responsible for detecting context updates, and consequently requesting the necessary adaptations, authorizations, etc for applying on the content.

Finally, the Content Server stores not only the content, but also the associated metadata.

\section{VIDEO SURVEILLANCE APPLICATION SCENARIO}

In our particular example scenario, illustrated in Figure 2, video surveillance is used as the primary means of a deterrent measure against unauthorized access to, for example, a car manufacturing plant. In addition to the video surveillance system operated by numerous strategically positioned cameras continuously monitoring both inside and outside of the plant, the surveillance relies on a team of security guards with mobile monitoring devices (i.e., dedicated Personal Digital Assistants (PDAs)), and a control room, in which the collected data is processed, compressed and stored in the highest possible resolution, bit/frame rate and quality for further use if needed. The guards are expected to have varying levels of access to different parts of the manufacturing plant, as the premises of the research and development and marketing strategy departments may be protected at a higher level than other parts of the site, which are housing the administrative offices, canteen areas, etc. In this way, while some senior guards can access video content from a number of positions, the junior guards are only able to receive content related to their permitted areas.

This application scenario is particularly significant, as it involves the co-existence of both realtime and stored compressed video content acquired through the surveillance cameras. The video feeds are of encrypted nature, so as to avoid intervention by unauthorized access to the captured content, which requires the protection of not only the content itself, but also the metadata through encryption. Authentication is important, as it ensures the integrity of content via means of protection, while also providing the necessary proof that an authentic source has generated the content. Relevant licenses are used while accessing the surveillance content, as they help to associate different qualities and levels of access to the same content among the users, as discussed above. Privacy features are needed, as different regions of the surveillance content can be protected in a different way to protect the privacy of captured particular subjects in a scene by, for instance, scrambling faces, etc. In this scenario, a two-level encryption scheme is considered. Each piece of content is encrypted using a random encryption key (e.g., key $\mathrm{x}_{1}$ in Figure 2), and in the same way, metadata is encrypted using another random key (e.g., key $\mathrm{x}_{2}$ in Figure 2). These random keys are in turn encrypted with whichever keys afford access to particular pieces of data (e.g., keys $A_{x}, B_{x}$, or $C$ in Figure 2). For example, $A_{x}$ could be used to encrypt $x_{2}$ and $B_{x}$ to encrypt $x_{1}$ and $x_{2}$. In this way, $A_{x}$ would only be supplied to entities allowed to access the metadata, but not the content, such as untrusted AEs; and $\mathrm{B}_{\mathrm{x}}$ would only be supplied to trusted AEs and authorized users. 


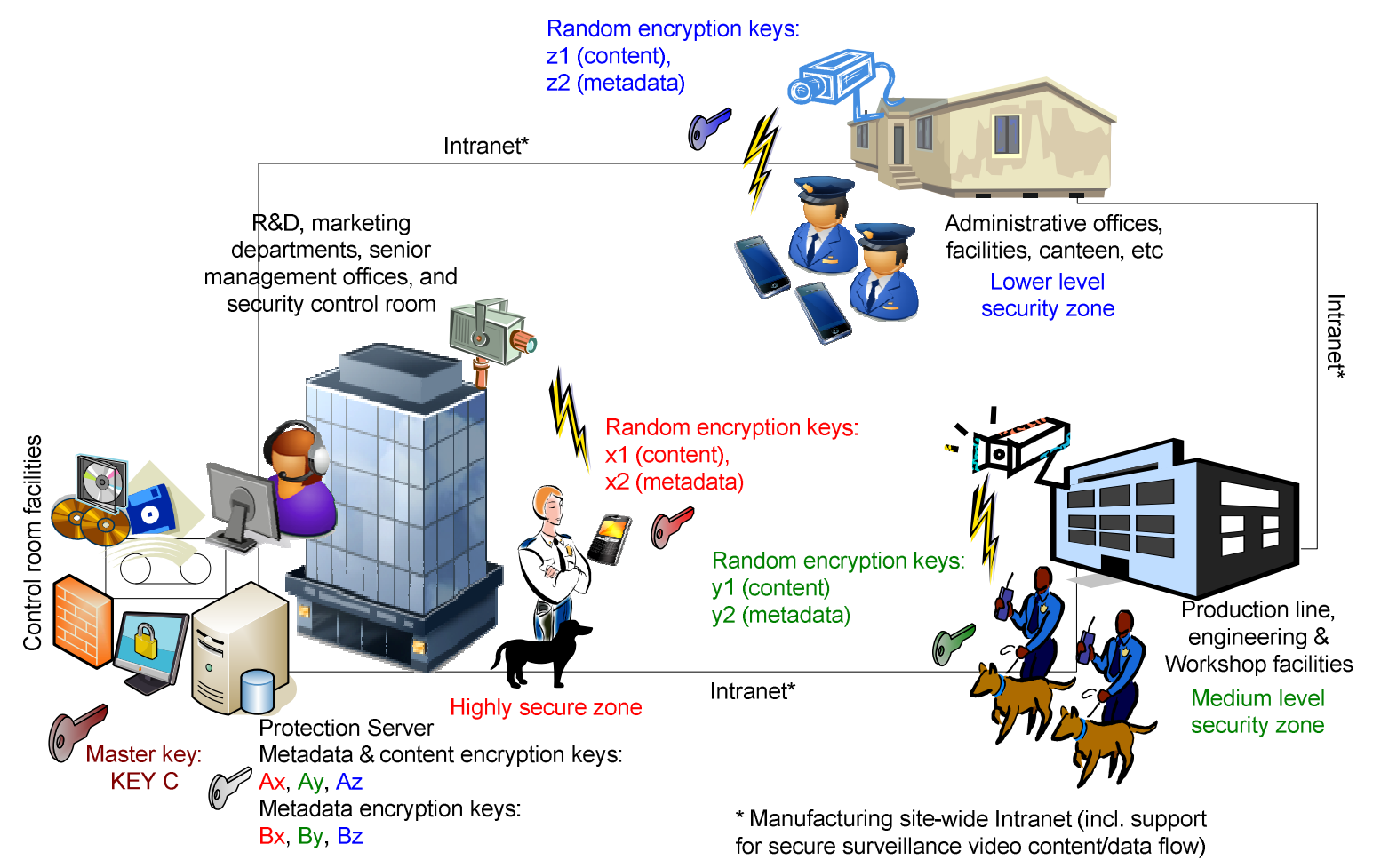

Fig. 2. Video Surveillance application scenario

It is equally important that the surveillance video content can be adapted, as both mobile security team guards and members of the fixed control unit staff may wish to access the same content with varying terminal features and access network technologies as well as under diverse usage environment conditions, including their preferences towards different portions of the captured live or stored video information. All of these necessitate a set of tools for adapting secured surveillance content, such as transrating, cropping and focusing into an area of attention, summarization of stored video, etc, in such a complex and heterogeneous yet governed multimedia application scenario.

The subsequent subsections will provide further descriptions of the two specific use cases identified within the aforementioned application scenario along with the associated requirements, and work and parameter exchange flows that are envisaged to prevail in these use cases in detail.

\section{A. Use case $1-$ Guards moving around buildings and receiving the surveillance content on their PDAs}

The first use case in the Video Surveillance application scenario focuses on patrolling security guards on site (both inside and outside of the buildings). The guards are equipped with PDAs, dedicated to receive both on-line (i.e., live) and off-line (i.e., recorded) compressed surveillance content through the existing wireless access medium, such as a WiMAX network set-up on and 
around the premises. The content is captured through numerous surveillance cameras deployed across the site, and data from these cameras is collected by the main processing unit in the control facility, which is then compressed and distributed to the PDAs of the requesting guards based on their location.

In this use case, the authenticated surveillance content is secured through encryption with a random key (e.g., key $\mathrm{y}_{1}$ ) and similarly, the related metadata is encrypted with another random key (e.g., key $\mathrm{y}_{2}$ ), which are used to encrypt every piece of data; and the associated licenses restrict access to the secured content and the related metadata depending on the location of the guards as well as their authorized access levels. The guards are expected to possess varying levels of access to different parts of the manufacturing plant; and this can be arranged using their staff membership identifiers. Therefore, there is a need for a number of encryption keys (i.e., key $\mathrm{A}_{\mathrm{y}}$, key $\mathrm{B}_{\mathrm{y}}$ ), and associating identifiers, which are related to the staff access levels. These keys are used to encrypt the content and metadata encryption keys (e.g., key $\mathrm{y}_{1}$ and $\mathrm{y}_{2}$, respectively). The different encrypted versions of the random encryption keys are stored in the Protection Server; every time a guard moves from one area to another, he/she needs to be re-authorized to view any content, and if so, the Governance Server supplies him/her with the necessary key(s), previously acquired from the Protection Server (e.g., key $A_{y}, B_{y}$ ).

All of the keys referred above can be used with a fast and secure symmetric encryption algorithm like Advanced Encryption Standard (AES). Current software implementations of this algorithm can cope with real-time encryption of video data at the bit rates that are usual for mobile devices. Depending on the security requirements different key lengths can be used, e.g., 128-bit for bulk-encryption keys $\left(\mathrm{y}_{1}, \mathrm{y}_{2}\right)$ and 192-bit for key-encryption keys $\left(\mathrm{A}_{\mathrm{y}}, \mathrm{B}_{\mathrm{y}}\right)$.

As the usage environment of the surveillance content primarily involves access through a mobile and wireless network by the end-terminals with restricted capabilities and display sizes (i.e., PDAs in this particular use case), it is necessary to provide low resolution and low bit/frame rate version of the original rich video (with the highest possible resolution, bit/frame rate and quality) to serve for the prevailing limited network bandwidth and device display size constraints. This is achievable through employing adaptation capabilities of the proposed architecture on the scalable video captured by the surveillance system. Furthermore, a minimum limit for the content resolution is guaranteed through the use of licenses for the guards to receive the necessary quality of the video to be able to detect any incident. In this way, a preview quality video can be fed to the mobile guards unless further and more specific adaptation operations are authorized for a particular guard and his/her terminal.

Typically, the operation of the architecture in this particular use case can be as follows: a mobile security guard logs into the surveillance system through his/her PDA (i.e., accessing via the Supervision Server) and tries to access the content being recorded in a certain surveillance area. The Intermediary detects that the captured and recorded video content occupies too much space due to its very large size, and realizing that the user requests it through his/her mobile terminal over a resource-limited wireless access network, it directly contacts the AE with a 
request for downscaling the large size of the requested video for a suitable viewing condition. While doing this, it is expected to supply the AE with the relevant context (not encrypted) of the usage environment, such as the terminal display size, network conditions, security IDs, etc (MPEG-21 DIA Usage Environment Description (UED) descriptors).

At this stage, an authorization for such adaptation also needs to be obtained. The Governance Server supplies the AE with the necessary information on the permitted adaptations. If the AE is an untrusted one, it performs adaptation of the content without the need for decrypting it (the Governance Server will allow the adaptation, but will only attach the metadata decryption key (e.g., key $\mathrm{A}_{\mathrm{y}}$ ) within the Adaptation Authorization Response). Otherwise (i.e., trusted), the key associated to content and metadata (e.g., key $\mathrm{B}_{\mathrm{y}}$ ) will be supplied by the Governance Server, as usual through the Supervision Server. During these operations, if the guard, who has requested the surveillance content, moves to an "untrusted" area, the context update associated to the new environment is supplied to the Governance Server, which then contacts the Content Server to interrupt the streaming of the secured surveillance video.

If the guard tries to continue accessing the protected content from the untrusted area or even from outside the system, the access will be prevented by a number of measures. First, access is only permitted through a trusted client, which will not make the decryption keys available to external applications, i.e., it will not be possible to extract and keep a local copy of the keys. Also, the authorization-based license enforcing mechanism provided by the Governance Server will not allow access to the protected content if the license conditions are not met. Finally, the security policy of the system may determine how often the encryption keys are changed, e.g., everyday or even every few minutes, in order to further hamper unauthorized access in case the trusted client was somehow bypassed.

The relevant parameter exchanges between the modules of the proposed architecture for this use case are depicted in Figure 3. 


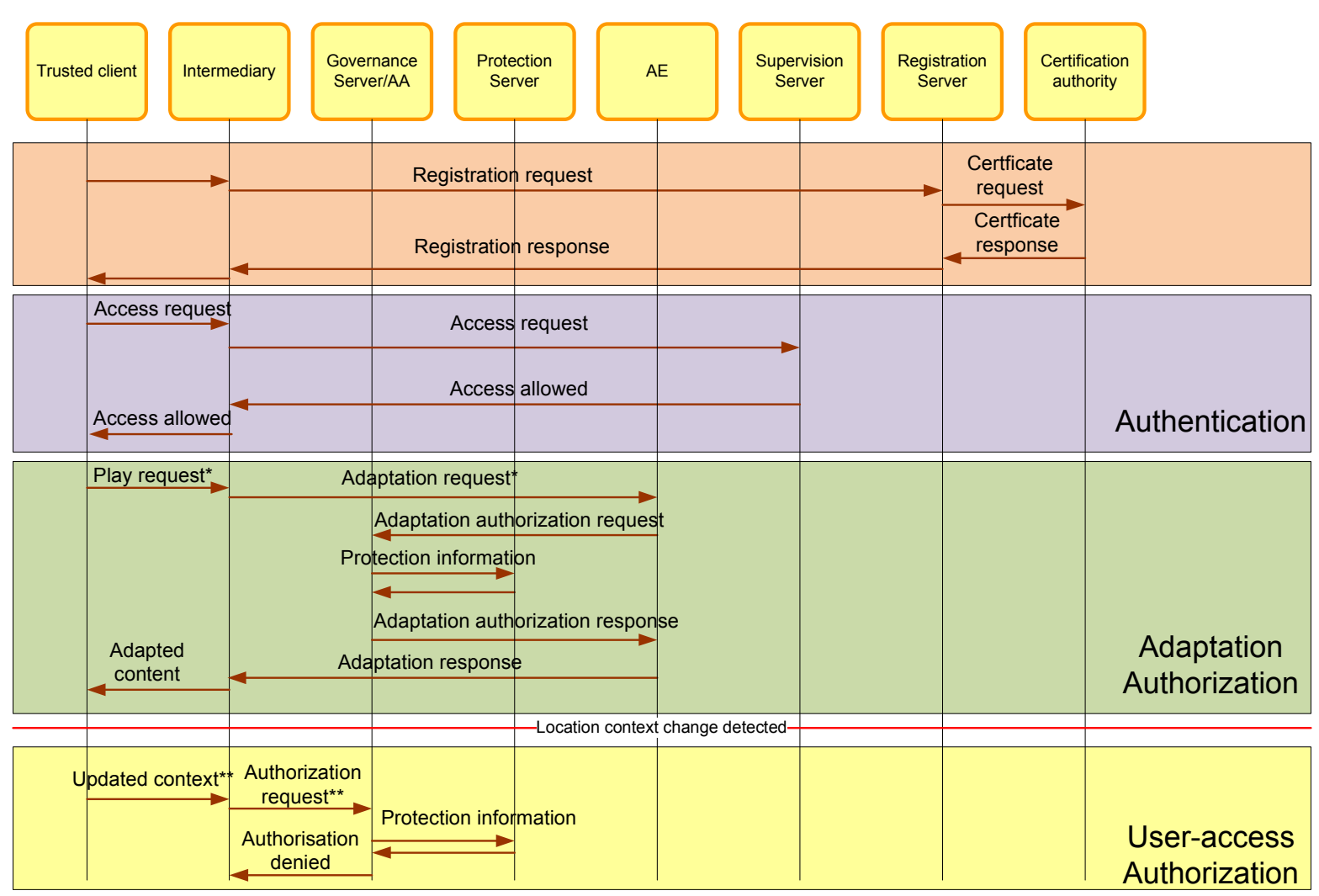

\footnotetext{
* User is in a "trusted" area
** User is now in an "untrusted" area
}

Fig. 3. Parameter exchanges between the modules of the proposed architecture for use case 1

\section{B. Use case 2 - Police investigation requires access to the recorded content and zoom into areas of attention in a scene in order to recognize faces}

In the second use case, the original content, which was captured and stored in the highest possible resolution, bit/frame rate and quality, is accessed by the top level authorized users (e.g., the head of the security team on site, the police, etc) later, so as to investigate a potential security breach that has been reported following an incident. Thus, the highest resolution content is encrypted with different random keys (e.g., key $\mathrm{x}_{1 \mathrm{a}}, \mathrm{x}_{1 \mathrm{~b}}$, etc) for each part of the content, which has a different access level. All of these content encryption keys are encrypted with the master key (i.e., key C). In order to restrict access to specific parts of the secured content, keys $\mathrm{x}_{1 \mathrm{a}}, \mathrm{x}_{1 \mathrm{~b}}$, etc are also encrypted with other encryption keys, such as keys $\mathrm{B}_{\mathrm{xa}}\left(\right.$ for $\left.\mathrm{x}_{1 \mathrm{a}}\right), \mathrm{B}_{\mathrm{xb}}\left(\right.$ for $\mathrm{x}_{1 \mathrm{a}}$ and $\mathrm{x}_{1 \mathrm{~b}}$ ), etc. Then, depending on his/her role and location, the appropriate decryption key (key $\mathrm{B}_{\mathrm{xa}}$, key $\mathrm{B}_{\mathrm{xb}}$, key $\mathrm{C}$, etc) will be delivered to the user. Moreover, associated content-related metadata information is encrypted using a random key (e.g., key $\mathrm{x}_{2}$ ), which is in turn encrypted with keys $A_{i}$ and $B_{i}$ (where $i$ stands for $x_{a}, x_{b}$, etc). Metadata is also stored and encrypted (as detailed in use 
case 1), and contains information related to time stamps, location, camera angle identification, etc of the captured video feed along with the necessary licenses for accessing the content.

As in use case 1, the keys mentioned above will belong to a symmetric encryption algorithm like AES. In this case, more sensible data can be encrypted with stronger keys, e.g., a 192-bit key can be used for high resolution content, and a 256-bit key can be used as the master key (key C).

As discussed before, licenses are required for associating different qualities and levels of access to the content, and hence also related to the metadata. Furthermore, the relevant metadata assists the authentication tasks to ensure the integrity of the content is preserved, while also providing a proof that an authentic source has generated the content. Event reporting information is also stored in a repository, and the Supervision Server provides functionalities to users for consulting the summaries of the reports, etc.

If a brief operational workflow is drawn as per the description provided in the previous use case, it can be exemplified as follows: upon receiving an alert, a police officer logs into the surveillance system (via the Supervision Server) through a Personal Computer (PC) located in the control room and selects to view a specific portion of the content recorded by one of the surveillance cameras. The Governance Server checks if the user has the appropriate permissions to view the requested video content, and as he/she is a member of the police, the video is rendered. For this purpose, the Governance Server supplies the decryption key to the Intermediary by requesting it from the Protection Server following the positive authorization of the user. The user's terminal is either the PC monitor or a TV screen with high resolution capabilities, as opposed to the PDA terminals used by the mobile security guards, as per in the first use case. The request from the police user includes not only the video content, but also the summary of the Event Reports related to the video and the metadata (e.g., time, place, recording camera, etc). The police officer selects a specific region of a scene and tries to zoom into, and thus focus on a suspicious looking person or persons in this scene in order to recognize the face(s). This request indicates the need for an adaptation operation on the stored high resolution surveillance content. Thus, the Governance Server checks whether the user can perform this adaptation and given that he/she has the appropriate permissions, the image is adapted and rendered. For performing this operation, the Governance Server needs to supply the decryption key to the $\mathrm{AE}$, as the particular adaptation request requires further accessing into the low-level and possibly semantic and structural specifics of the compressed video content. The associated parameter exchanges between the modules of the proposed architecture for this use case are depicted in Figure 4. 


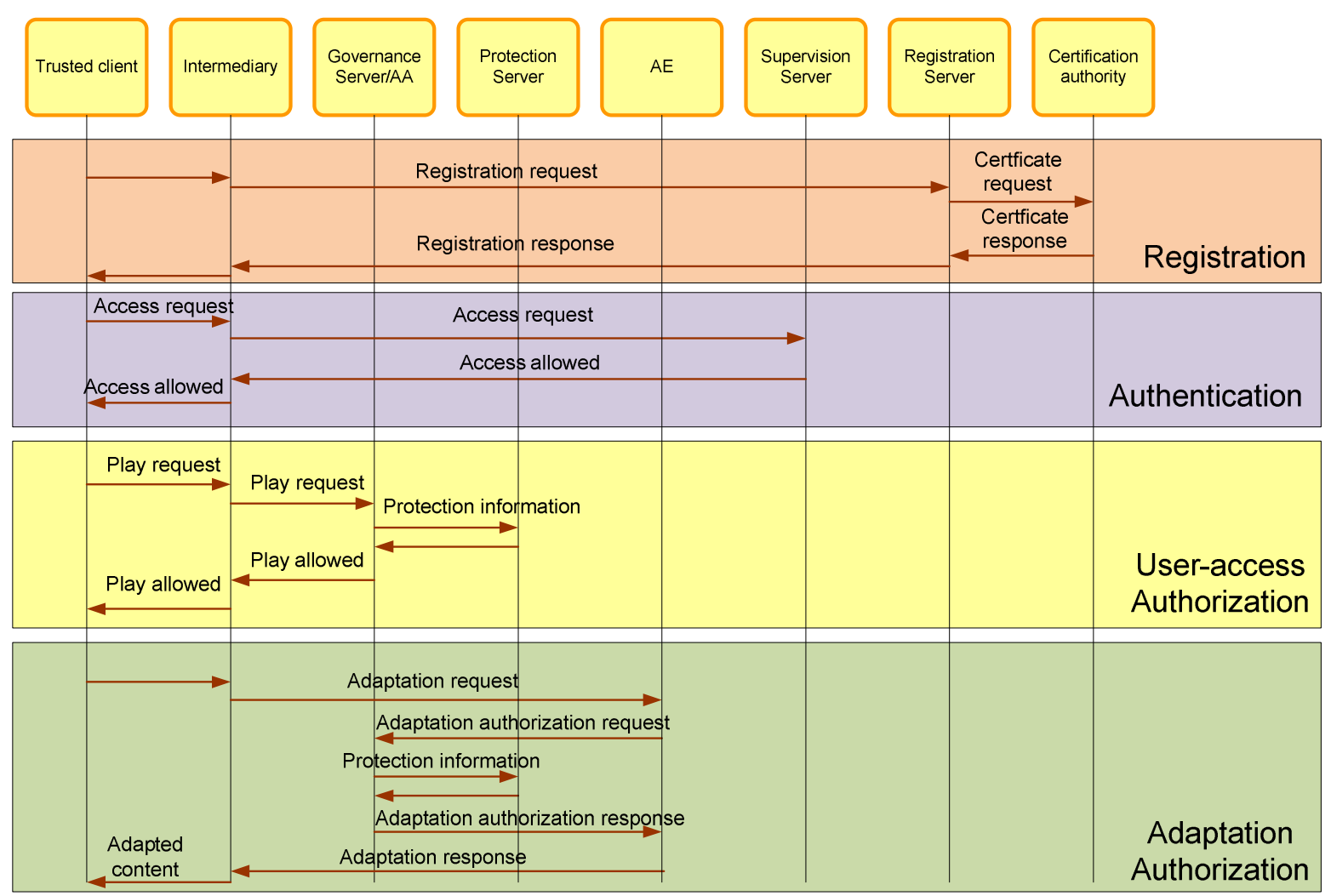

Fig. 4. Parameter exchanges between the modules of the proposed architecture for use case 2

\section{RESULTS AND DISCUSSIONS}

Governance Server, not only copes with the "traditional" authorization, which accepts or denies a particular user the access to a particular content, but also deals with the governance of various types of adaptations by means of fine-grained descriptions (expressed in MPEG-21 DIA) integrated in MPEG-21 licenses.

In use case 1, for example, when a junior guard located in a "trusted" area would try to access the surveillance content, an automatic adaptation request would be sent from the Intermediary to the AE. The subsequent authorization request message sent by the AE to the Governance Server is shown in Figure 5. The message specifies the membershipID, the resourceID, the right requested by the user, and his/her location; and as can be seen from this figure, it is expressed based on the MPEG-21 REL and MPEG-7 descriptors to guarantee interoperability.

The same authorization request could also be used for use case 2 . The only differences in this case would be that the Principal would be a police officer (and thus, the user identifier would be different from that of Figure 5), the resource would be pieces of recorded content from several rooms (e.g., "urn:videosurveillance") or a specific region of interest of the recorded content of a particular room (e.g., "urn:videosurveillance:D6:room008:ROI10"), and the location would be 
the Security Control Room instead of the one mentioned in Figure 5, which would be a "highly secure" zone.

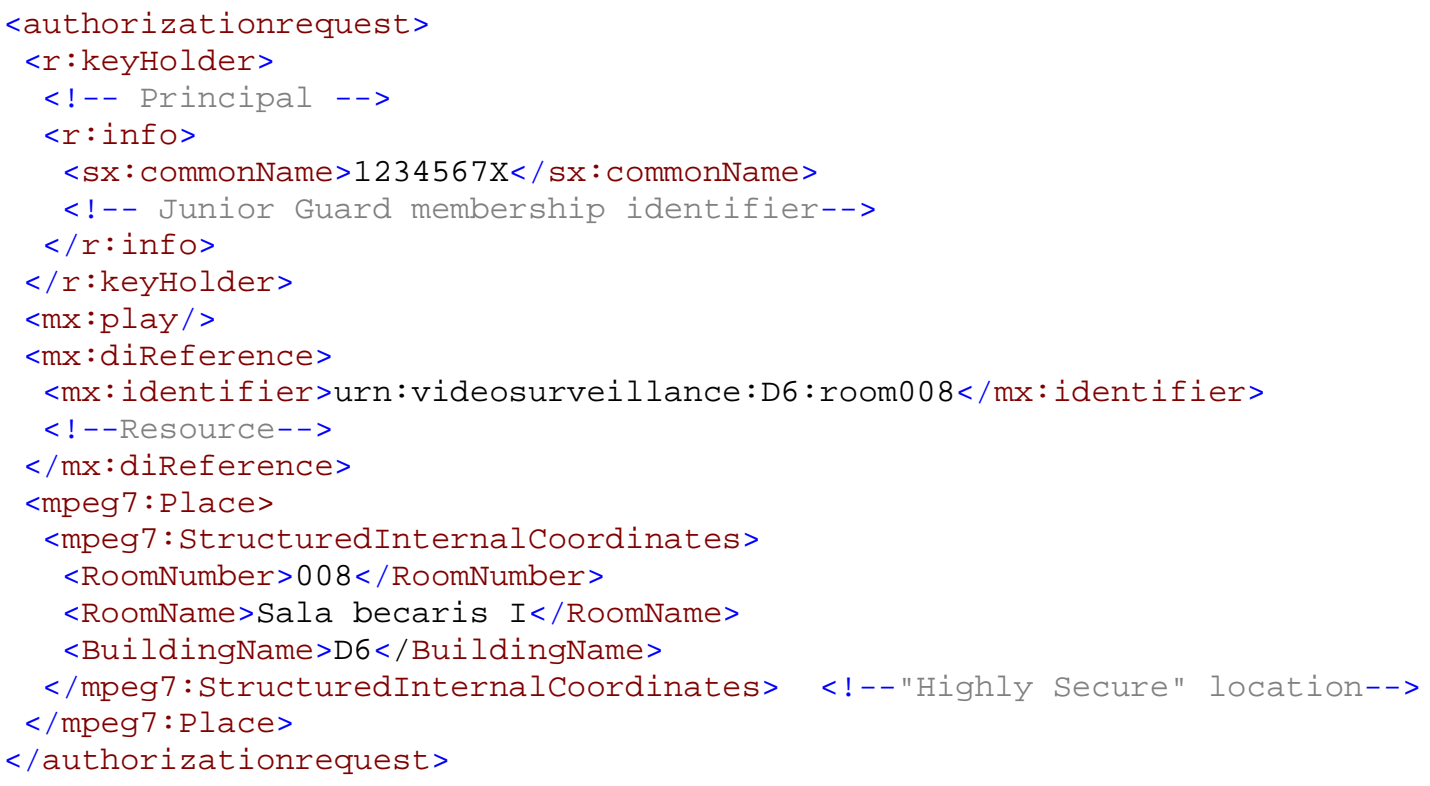

Fig. 5. Authorization request example

The Governance Server would, then, check the repository of licenses in order to identify the licenses associated to the required resource and principal, and subsequently would or would not authorize the request by comparing the information of the authorization request message with the conditions specified in the license. As can be seen in Figure 6, the authorization response message includes an authorization response element, which is a Boolean, a list of the permitted adaptations and their corresponding constraints specified in the license, and the necessary key to decrypt the content and/or metadata encryption keys. 


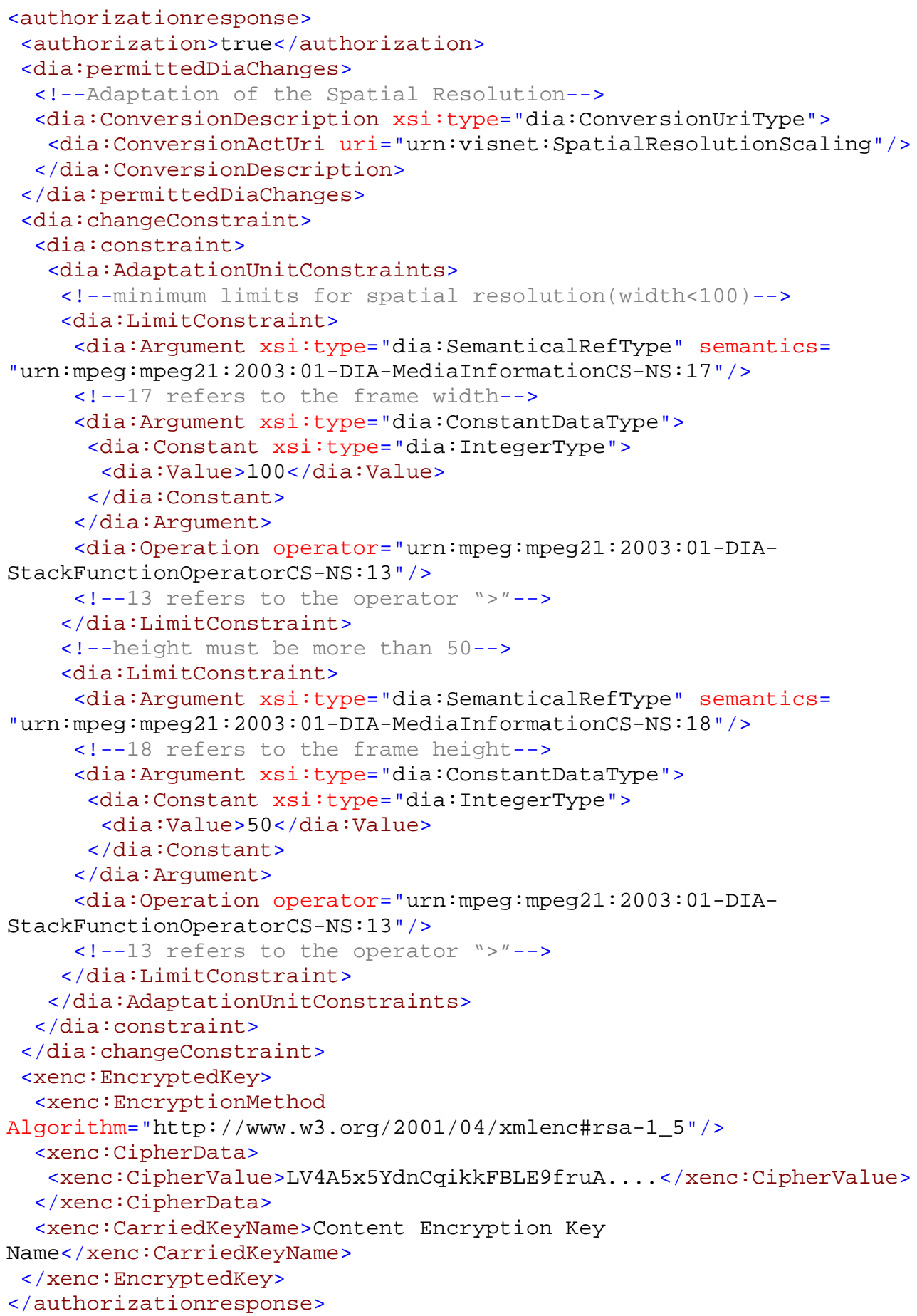

Fig. 6. Authorization response example 
It is also a remarkable fact that to date, the MPEG-21 REL "Location" element has merely allowed expressing a postal address; and hence, we believe that for the Video Surveillance application scenario, it is essential that the conditions included in the licenses should be able to express more meaningful and accurate locations, such as Global Positioning System (GPS) coordinates or any other internally structured coordinates. Thus, we propose to extend the MPEG-21 REL location element with the MPEG-7 "Place" element. We envisage that this extension should be a part of a new MPEG-21 REL profile for Video Surveillance applications.

Regarding the user-access authorization (Figure 4), the exchange of messages would be the same. The Intermediary would be responsible for guaranteeing that the user access is performed respecting the adaptation constraints (which should be fulfilled whether the content is adapted or not), and if necessary, for requesting the required adaptation operation from the AE.

In use case 1, the video content sent from the Content Server is downscaled by the AE. Since the video content has been encoded in a scalable format, this adaptation can be carried out by simply dropping parts of the content. Moreover, the video content is encrypted using the technology presented in [12], which allows adapting the encrypted contents without decrypting them. As a result, it is possible to use an untrusted AE to perform this adaptation. In this case, the $\mathrm{AE}$ only needs the access to the metadata for deciding the appropriate adaptation that satisfies the usage environment constraints such as terminal capabilities and network conditions. The input to the adaptation process is an encrypted stream. The AE selects which fragments of this stream are to be sent to the guard's terminal. The output is another stream, which is also encrypted, as no decryption is performed, and of a suitable size for the terminal to comfortably display (after being decrypted with the appropriate key). Table I summarizes the example usage environment constraints and related adaptation results. Visual comparison between the original and adapted sequences is shown in Figure 7. Please note that the Pedestrian high definition test video sequence has been used for demonstration purposes.

Table I. Example usage environment constraints and adaptation results for use case 1

\begin{tabular}{|c|c|}
\hline \multicolumn{2}{|c|}{ Constraints } \\
\hline Terminal display resolution & VGA (640×480)@15fps \\
\hline Channel bandwidth & $512 \mathrm{kbps}$ \\
\hline \multicolumn{2}{|c|}{ Original content } \\
\hline Spatial resolutions & $480 \times 256,960 \times 512$, and $1920 \times 1024$ pixels \\
\hline Temporal resolutions & $6.25,12.5$, and $25 \mathrm{fps}$ \\
\hline Quality levels per spatial resolution & 2 (Low and High) \\
\hline Bit rate & $5 \mathrm{Mbps}$ \\
\hline \multicolumn{2}{|c|}{ Adapted content } \\
\hline Spatial resolutions & $480 \times 256$ pixels \\
\hline Temporal resolutions & 6.25 and $12.5 \mathrm{fps}$ \\
\hline Quality of the highest resolution layer & Low \\
\hline Bit rate & $280 \mathrm{kbps}$ \\
\hline
\end{tabular}




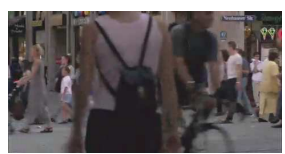

(a)

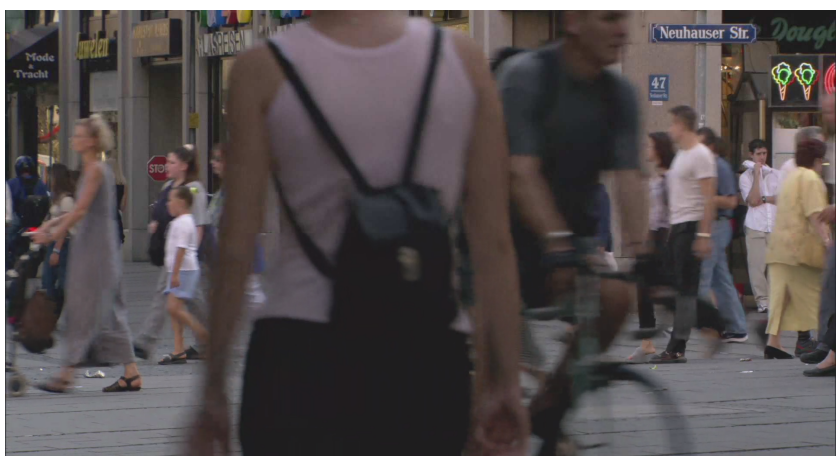

(b)

Fig. 7. The decoded bit streams (scaled to $13 \%$ of the original resolution): (a) adapted by dropping the highest spatial scalability layers and (b) original

In use case 2 , content is adapted upon request by a security officer in order to zoom into a specific region of a video, in this case to view with the highest possible detail of a person's face. In this scenario, we can consider that one type of adaptation is already being performed, namely the downscaling of the video to match the display resolution. After requesting to zoom into the region of interest, downscaling is no longer appropriate, which implies that the higher quality layers must be decrypted by means of the appropriate key. Instead, a new adaptation is applied: the cropping of the Region Of Interest (ROI). As with the previous use case, if this adaptation can be performed by dropping parts of the video stream, it is still possible to use an untrusted AE. In this case, the encoding of the original video content should be performed compatible to the needs of this type of adaptation (such as enabling the interactive ROI feature in the encoding stage). Furthermore, the pertinent metadata should be available to the untrusted AE. Nevertheless, the adaptation is performed by a trusted AE in our experiments, as the original video stream does not support the aforementioned feature. Therefore, the keys for decoding both the metadata as well as video data are received by the AE. As in the previous case, the AE uses the usage environment constraints to determine the properties of the adapted video such as the cropping window definition, output resolution and bit rate. Subsequently, the content is transcoded accordingly. Table II summarizes the example usage environment constraints and corresponding adaptation results.

Assuming that the encircled person standing in front of the shop is the ROI, Figure 8 (a) shows a snapshot of the adapted content. Moreover, Figure 8 (b) shows a snapshot of the video stream adapted using an alternative adaptation approach, which is the adaptation performed in use case 1. 
Table II. Example usage environment constraints and adaptation results for use case 2

\begin{tabular}{|l|l|}
\hline \multicolumn{2}{|c|}{ Constraints } \\
\hline Terminal display resolution & XVGA $(1024 \times 768) @ 60 \mathrm{fps}$ \\
\hline Channel bandwidth Original content \\
\hline \multicolumn{2}{|c|}{$1 \mathrm{Mbps}$} \\
\hline Spatial resolutions & $480 \times 256,960 \times 512$, and $1920 \times 1024$ pixels \\
\hline Temporal resolutions & $6.25,12.5$, and $25 \mathrm{fps}$ \\
\hline Quality levels per spatial resolution & 2 (Low and High) \\
\hline Bit rate & $5 \mathrm{Mbps}$ \\
\hline \multicolumn{2}{|c|}{ Adapted content } \\
\hline Spatial resolution & $960 \times 512$ pixels \\
\hline Temporal resolution & $25 \mathrm{fps}$ \\
\hline Quality of the highest resolution layer & $\begin{array}{l}\text { Standard (The adapted sequence is a non- } \\
\text { scalable video stream) }\end{array}$ \\
\hline Bit rate & $970 \mathrm{kbps}$ \\
\hline
\end{tabular}

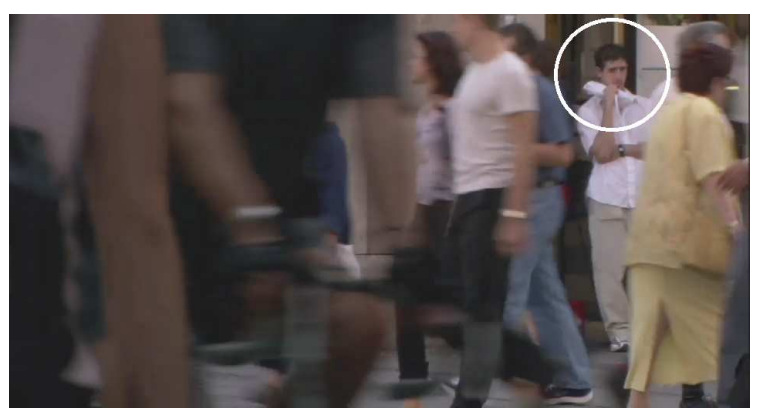

(a)

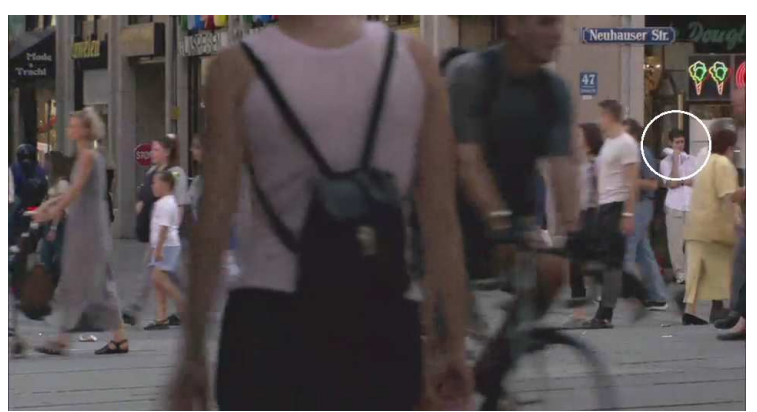

(b)

Fig. 8. The decoded bit streams (scaled to $26 \%$ of the original resolution): (a) adapted by cropping the ROI and (b) adapted by dropping the highest spatial scalability layers

\section{CONCLUSIONS AND FUTURE WORK}

This paper has presented a proposed architecture for the secure adaptation and distribution of digital contents in governed multimedia applications. This is a modular architecture, which enables the adaptation of any kind of secured content to enhance users' perceived quality in heterogeneous usage environments. The proposed functional architecture can be used to manage protected contents in a wide variety of applications, ranging from collaborative scenarios to security applications; from infotainment and e-services to numerous prosumer applications. This paper has focused on Video Surveillance applications, because they are present in our everyday 
lives, and they pose a challenge for addressing privacy protection and the secure management of digital content in heterogeneous usage environments. Due to the diverse nature of the scenario under consideration, two different use cases have been presented.

Further work in this area may consider a variation of the second use case, where most of the video is encrypted with one key, yet some sensitive parts are encrypted with a different and more restrictive key, for which a special license is required. For example, in order to protect users' privacy, the faces of persons appearing in the video can be scrambled with a higher level key, while the rest of the scene is scrambled with a lower level key. Consequently, the guards would then have access to the latter part of the overall video, and would be able to view the protected content except for the faces in the image. The authorized police officers only would be able to use the higher level key to zoom into a specific ROI and view this particular region in detail.

\section{ACKNOWLEDGMENT}

The work presented was developed within VISNET II, a European Network of Excellence (http://www.visnet-noe.org), funded under the European Commission IST FP6 programme.

\section{REFERENCES}

[1] E. Rodríguez, "Standardisation of the protection and governance of multimedia content," Ph.D. Thesis, Department of Information and Communication Technologies, Universitat Pompeu Fabra, Barcelona, 2006.

[2] F. López, J. M. Martínez, and N. García, "A model for preference-driven multimedia adaptation decision-making in the MPEG-21 framework," Multimedia Tools and Applications, March 2010, ISSN 1380-7501 (In print).

[3] A. Vetro and C. Timmerer, "Digital item adaptation: overview of standardization and research activities," IEEE Trans. Multimedia, vol. 7, no. 3, Jun. 2005.

[4] A. Carreras and J. Delgado, "A new type of contextual information based on the adaptation authorisation," in Proc. $9^{\text {th }}$ Int. Workshop on Image Analysis for Multimedia Interactive Services (WIAMIS 2008), Klagenfurt, Austria, 7-9 May 2008.

[5] S. J. Wee and J. G. Apostolopoulos, "Secure scalable streaming and secure transcoding with JPEG-2000," in Proc. IEEE Int. Conf. Image Processing, Sep. 2003.

[6] D. Engel, T. Stütz, and A. Uhl, "Format-compliant JPEG2000 encryption in JPSEC: security, applicability and the impact of compression parameters," EURASIP J. Information Security, vol. 2007, no. 8, Jan. 2007.

[7] C. Bergeron and C. Lamy-Bergot, "Compliant selective encryption for H.264/AVC video," in Proc. IEEE 7th Workshop on Multimedia Signal Processing, Shanghai, China, Oct. 2005.

[8] Z. Shahid, M. Chaumont, and W. Puech, "Fast protection of H.264/AVC by selective encryption of cabac," in Proc. IEEE International Conference on Multimedia and Expo, Cancun, Mexico, Jun. 2009.

[9] S. Spinsante, F. Chiaraluce, and E. Gambi, "Masking video information by partial encryption of H.264/AVC coding parameters," in Proc. 13th European Signal Processing Conference, Antalya, Turkey, Sep. 2005. 
[10] M. Grangetto, E. Magli, and G. Olmo, "Conditional access to H.264/AVC video by means of redundant slices," in Proc. 2007 IEEE International Conference on Image Processing, Texas, USA, Sep. 2007.

[11] T. Stütz and A. Uhl, "Format-compliant encryption of H.264/AVC and SVC," in Proc. IEEE International Symposium on Multimedia, Berkeley, California, USA, Dec. 2008, pp. 446-451.

[12] H. Kodikara Arachchi, X. Perramon, S. Dogan, and A.M. Kondoz, "Adaptation-aware encryption of scalable H.264/AVC video for content security," Elsevier J. Signal Processing: Image Communication-Special Issue on Scalable Coded Media beyond Compression, Vol. 24, No. 6, Jul. 2009, pp. 468-483.

[13] H. Hellwagner, R. Kuschnig, T. Stütz, and A. Uhl, "Efficient in-network adaptation of encrypted H.264/SVC content," Elsevier J. Signal Processing: Image Communication, Vol. 24, No. 9, Oct. 2009, pp. 740-758.

[14] N.M. Thomas, D.R. Bull, and D.W. Redmill, "A novel H.264 SVC encryption scheme for secure bit-rate transcoding," in Proc. IEEE Picture Coding Symposium, Chicago, USA, May 2009.

[15] D. Mukherjee, H. Wang, A. Said, and S. Liu, "Format independent encryption of generalized scalable bit-streams enabling arbitrary secure adaptations," in Proc. IEEE Int. Conf. Acoustics, Speech, and Signal Process., Philadelphia, PA, USA, Mar. 2005.

[16] V. Torres, J. Delgado, et al., "An implementation of a trusted and secure DRM architecture," in On the Move to Meaningful Internet Systems 2006: OTM 2006 Workshops (IS'06), Lecture Notes in Computer Science, vol. 4277. Springer-Verlag, 2006, 312-321.

[17] J. Delgado, V. Torres, S. Llorente, and E. Rodríguez, "Rights management in architectures for distributed multimedia content applications", in 21st Tyrrhenian Workshop on Digital Communications: Trustworthy Internet, Ponza, Italy, Sep. 2010; http://dmag.ac.upc.edu/ downloads/papers/jdvtslerTWDCTI.pdf. 